\title{
La justice sociale dans la construction du jugement d'acceptabilité. Analyse des réactions d'agriculteurs face à différentes règles de partage de l'eau souterraine
}

Social justice in the construction of the acceptability judgment. Analysis of the farmers' reactions to different groundwater sharing rules

Clémence Moreau, Jean-Daniel Rinaudo et Patrice Garin

\section{CpenEdition Journals}

\section{Édition électronique}

URL : http://journals.openedition.org/economierurale/4612

DOI : 10.4000/economierurale.4612

ISSN : 2105-2581

Éditeur

Société Française d'Économie Rurale (SFER)

Édition imprimée

Date de publication : 15 mars 2015

Pagination : 31-48

ISSN : 0013-0559

Référence électronique

Clémence Moreau, Jean-Daniel Rinaudo et Patrice Garin, « La justice sociale dans la construction du jugement d'acceptabilité. Analyse des réactions d'agriculteurs face à différentes règles de partage de l'eau souterraine », Économie rurale [En ligne], 346 | Mars-avril 2015, mis en ligne le 15 mars 2017, consulté le 01 mai 2019. URL : http://journals.openedition.org/economierurale/4612 ; DOI : 10.4000/ economierurale. 4612 


\title{
La justice sociale dans la construction du jugement d'acceptabilité Analyse des réactions d'agriculteurs face à différentes règles de partage de l'eau souterraine
}

\author{
Clémence MOREAU, Jean-Daniel RINAUDO • Bureau de Recherches Géologiques et Minières \\ (BRGM), Unité Nouvelles Ressources et Économie, Montpellier \\ jd.rinaudo@brgm.fr. \\ Patrice GARIN • Irstea, UMR G-Eau, Montpellier
}

Cet article analyse les discours des agriculteurs en réaction à différents modes de calcul de quotas individuels, pouvant être utilisés pour la mise en place de la loi sur l'eau de 2006. La démarche consiste à articuler deux types d'approches : I'évaluation de l'acceptabilité de ces règles et l'analyse des principes de justice sociale sous-jacents aux argumentaires. Cette problématique est abordée à travers une revue de la littérature et une étude empirique, consistant à soumettre neuf scénarios d'allocation à 76 agriculteurs sélectionnés dans cinq terrains en France. Cet article contribue à la littérature sur la justice sociale dans la gestion de l'eau, en combinant différents cadres d'analyse ; il donne également des outils pour la mise en place d'une gestion quantitative, en proposant une méthode d'évaluation a priori de l'acceptabilité des modes de calculs des quotas.

MOTS-CLÉS : acceptabilité, justice sociale, loi sur l'eau, politiques publiques, irrigation

Social justice in the construction of the acceptability judgment.

Analysis of the farmers' reactions to different groundwater sharing rules

This article analyses farmers' answers to different water allocation rules which could be used to implement the 2006 French Water Law. We develop an analytical framework which combines an evaluation of overall acceptability of these rules with an analysis of the underlying principles of social justice. This framework is applied through conducting a survey in five French case studies. Nine allocation rules are presented to 76 irrigating farmers and their preferences analyzed. This article contributes to literature on social justice by articulating different analysis framework, and gives tools to quantitative management implementation, by proposing an assessment method on calculation quotas acceptability. (JEL: Q25, Q15, Q28, Q54, K32, H39)

KEYWORDS: acceptability, social justice, framework water directive, public policy, irrigation

D epuis 1980, l'irrigation individuelle s'est fortement développée dans l'agriculture française, notamment à partir de forages individuels (Loubier et al., 2013). Le cadre institutionnel, initialement peu contraignant, s'est renforcé avec la loi sur l'eau de 1992, puis avec la loi sur les milieux aquatiques de 2006. Cette dernière impose de définir un volume prélevable pour les bassins et aquifères considérés comme intensément exploités. Dans ces zones, un volume dédié à l'agriculture est défini et sa gestion confiée à une structure, l'Organisme unique de gestion collective (OUGC), qui représente les intérêts des agriculteurs irrigants. Il est détenteur d'une autorisation unique de prélèvement qu'il est chargé de répartir entre ses adhérents (Figureau et al., 2012;2014). Globalement, les volumes alloués au secteur agricole sont réduits par rapport au niveau moyen de consommation historique. 
Cette évolution représente un « changement de paradigme » (Montginoul et al., 2011), remettant profondément en cause le rapport que le monde agricole avait établi avec cette ressource souterraine, marqué par un accès quasi libre et des mécanismes de gestion de crise de plus en plus fréquents ces dix dernières années. La loi sur l'eau de 2006 instaure un changement de pratiques, en permettant aux agriculteurs de connaître, avant le début de la culture, le volume d'eau dont ils disposent et d'ajuster leurs assolements en conséquence pour réduire le risque (technique et économique) lié aux interdictions d'arrosage en cours de saison (Martin, 2013). Mais elle implique également un changement cognitif : l'eau de nappe, perçue jusqu'alors comme abondante dans un grand nombre de bassins versants, devient rationnée. Désormais, la contrainte créant la rareté n'est plus d'ordre naturel (sécheresse, épuisement des ressources), mais administratif (respect d'un volume prélevable).

L'inquiétude générée par cette évolution au sein de la profession agricole est accentuée par l'incertitude relative aux règles qui détermineront la définition des quotas individuels. La loi de 2006 prévoit en effet que chaque organisme unique puisse établir ses propres règles en matière de répartition du volume prélevable, considérant qu'il est le mieux placé pour définir des règles adaptées sur le territoire. Pour les Organismes uniques, l'enjeu consiste donc à définir des règles de répartition acceptées et respectées par les agriculteurs, en période normale comme en période de crise ; et pour les agriculteurs, l'enjeu est de recevoir un volume individuel qui n'impacte pas négativement leur revenu et la valeur du foncier. Une hypothèse, avancée par d'autres équipes (Nancarrow et Syme, 2001, 2004 ; Syme et al., 1999 ; Syme et Nancarrow, 1997), est que la règle de répartition retenue sera d'autant plus acceptée que le partage de l'eau auquel elle conduit sera jugé juste. Cet article se propose de confronter cette hypothèse à la réalité de cinq terrains français. Il vise à mettre en évidence la multiplicité des conceptions de la justice sociale au sein du monde agricole, susceptible de compliquer l'obtention d'un consensus, et à comprendre comment s'opère l'agencement des références éthiques et des considérations plus pragmatiques (efficacité, facilité de mise en œuvre, etc.) dans les argumentaires des agriculteurs sur la répartition des eaux souterraines. Après avoir exposé le cadre conceptuel d'analyse d'acceptabilité des règles de répartition de l'eau, nous présenterons la méthodologie mise en œuvre, puis les résultats. Enfin, ces résultats sont discutés et mis en perspective.

\section{Cadre théorique pour analyser l'acceptabilité des règles d'allocation de l'eau}

Le principe de justice sociale est souvent invoqué dans les textes législatifs relatifs à la régulation de l'accès aux ressources en eau, mais rarement explicité, encore moins dans sa mise en œuvre opérationnelle (Syme et al., 1999 ; Movik, 2014 ; Roa-García, 2014). Comment donc définir ce qu'est une allocation juste de la ressource en eau ?

\section{Qu'est-ce que la justice sociale ?}

La littérature relative à la justice sociale apporte deux éléments de réponse. Le premier est la distinction entre justice distributive et justice procédurale (Alexander et Ruderman, 1987). La justice distributive concerne le résultat du processus d'allocation : il s'agit de qualifier le caractère juste de la répartition de la ressource entre les individus. La justice procédurale s'intéresse au processus décisionnel ayant conduit au choix d'une règle (représentativité, modalités d'arbitrages, etc.). Bien que les deux notions soient intimement liées, nous nous intéressons essentiellement à la justice distributive dans cet article. 
Le deuxième élément est qu'il n'existe aucune définition universelle de la justice, mais une pluralité de philosophies, toutes subjectives, car reflétant des positionnements éthiques et des principes intuitifs individuels. De nombreuses typologies de ces « philosophies de la justice » ont été proposées dans la littérature (Lamont et Favor, 1996 ; Wenz, 1988) ; elles peuvent être synthétisées en six principes majeurs.

- Le principe de stricte égalité. Chaque individu doit recevoir le même niveau de biens matériels et de service, en considérant que les hommes sont moralement égaux.

- Le principe d'égalité des chances. Est juste ce qui permet d'égaliser les chances d'accès aux fonctions et aux positions sociales : une répartition inégale des ressources peut parfois aider à accroître le bien-être global d'une société, mais elle n'est acceptable qu'à condition que l'inégalité de traitement bénéficie aux plus défavorisés.

- Le principe d'efficacité. Est juste ce qui est le plus efficace, c'est-à-dire qui permet d'optimiser le plus grand bien-être du plus grand nombre de personnes.

- Le principe d'antériorité. Un partage est juste s'il ne remet pas en cause la part des ressources que chacun a pu s'approprier légitimement dans le passé et si cette appropriation n'a dégradé le bienêtre de personne.

- Le principe de besoin. Une allocation est juste si elle procure les ressources de base nécessaires à la satisfaction des besoins essentiels des individus, dans le respect de leurs différences de situations.

- Le principe de mérite. Est juste une allocation qui repose sur une proportionnalité entre les montants reçus et les actions réalisées. Le mérite peut être mesuré en termes d'efforts consentis, ou selon les bénéfices pour la société.
Cette grille d'analyse est reprise par quelques auteurs étudiant les politiques d'allocations de l'eau sous l'angle de la justice sociale. Nous pouvons distinguer ici trois types d'analyses, selon qu'elles portent sur les principes philosophiques sous-tendant l'organisation générale de la gestion de l'eau dans un pays ; sur la mise en œuvre de ces principes à travers des politiques publiques; ou sur les règles établies localement par les usagers. Nous nous intéressons ici plus particulièrement à ce troisième type de travaux.

L'objectif est de mettre en évidence les facteurs qui déterminent le sentiment de justice, soit à travers une analyse a posteriori des règles d'allocation mises en œuvre, soit par une évaluation a priori des règles d'allocation présentées sous forme de scénarios. Cette dernière approche est largement mise en œuvre par Syme et Nancarrow. Ces auteurs fondent leur analyse sur des enquêtes auprès de représentants ou d'un public profane pour évaluer le niveau d'adhésion à diverses conceptions philosophiques de la justice distributive, présentées sous forme de courtes propositions qui reprennent dans un langage commun les principes énoncés précédemment. Par exemple : «En matière d'allocation d'eau, tous les usagers doivent être traités de la même manière »; "Toute l'eau devrait être allouée via les mécanismes de marché et vendue à ceux qui la paient le plus, sans considération pour l'usage qu'ils en font ». Les résultats montrent que les individus sont capables d'expliciter leur propre conception de la justice sociale et de se replacer intuitivement dans le cadre d'analyse présenté ci-dessus. Syme et Nancarrow prouvent cependant que le positionnement individuel peut varier selon le contexte de l'enquête. Si celle-ci est contextualisée, elle permettra de révéler une conception de la justice situationnelle, qui reflète les arbitrages que chaque individu réalise entre la défense de 
ses principes moraux, la défense de ses propres intérêts et des jugements sur la situation des autres personnes affectées. Une enquête plus décontextualisée fera au contraire émerger des conceptions de la justice restreintes aux valeurs morales et éthiques des individus, c'est-à-dire un jugement sur la justice universelle. Le choix de la méthodologie d'enquête retenue conditionne donc fortement le type de résultats obtenus.

Le processus de construction du sentiment de justice sociale fait l'objet de travaux en psychologie sociale, ayant conduit à l'émergence de la théorie de l'intuitionnisme, développée par Konow. Elle prend acte de tensions possibles entre principes éthiques chez toute personne qui, en situation, construit son sentiment de justice dans un compromis entre, par exemple, le principe d'efficacité de l'allocation des ressources, le principe de stricte égalité entre les attributaires et le principe de besoin, sous la contrainte de sa propre responsabilité. Même s'il ne catégorise pas les principes de justice selon une grille aussi détaillée que celle précédemment décrite, on retiendra que Konow évoque l'intuitionnisme de « sens commun », qui décrit comment les personnes interrogées réagissent spontanément face à une question de justice, en articulant des principes pouvant être jugés par ailleurs contradictoires.

\section{Les autres déterminants de l'acceptabilité}

D'autres travaux montrent que l'acceptabilité d'une nouvelle norme ou d'une innovation est déterminée par d'autres facteurs que la justice sociale, et notamment par leur légitimité et leur utilisabilité.

La légitimité comporte trois dimensions : normative, cognitive et réglementaire. La légitimité normative d'une règle renvoie à son fondement éthique, à l'adéquation entre les valeurs d'une règle et celles de la société, et fait écho aux principes qui fondent le sentiment de justice. La légitimité réglementaire implique que les institutions qui mettent en place des règles se conforment aux lois en vigueur dans la société. Enfin, la légitimité cognitive repose sur le système de significations et de symboles véhiculé par une règle, qui doit renvoyer à une représentation de la réalité compréhensible et acceptable pour les acteurs concernés. Ce cadre d'analyse est utilisé pour qualifier l'acceptabilité des mesures agro-environnementales à enjeu « eau » dans le Sud-Ouest de la France.

La question de l'utilisabilité est liée à la faisabilité des changements (techniques, institutionnels, sociaux, etc.) associés à une réforme. L'utilisabilité du nouveau mécanisme d'allocation sera évaluée par les acteurs concernés en termes de « compatibilité du système avec les pratiques, les ressources, les objectifs des usagersutilisateurs potentiels et de leur situation » (Dubois et Bobillier-Chaumon, 2009). L'utilisabilité peut ainsi être évaluée au regard de cinq critères (Nielsen, 1994) : l'efficience, la satisfaction subjective, la facilité d'apprentissage, la facilité d'appropriation et la fiabilité de la mesure.

\section{Articulation des notions de justice sociale et d'acceptabilité}

L'objectif de cet article consiste à articuler ces deux cadres d'analyse de l'acceptabilité et de la justice sociale, à travers une étude empirique réalisée en France. L'approche retenue a consisté à présenter à des irrigants un nombre limité de mécanismes d'allocation de l'eau, susceptibles de s'appliquer à eux dans un avenir proche. Les résultats obtenus ont été analysés en termes de justice situationnelle, et plus globalement, d'acceptabilité. Nous nous sommes limités à la problématique du partage de l'eau au sein du secteur agricole, l'allocation entre les secteurs (urbain, industriel, agricole et environnement) étant supposée résolue en amont. 


\section{Cas d'étude et méthodologie}

\section{Vue d'ensemble de la démarche}

La méthodologie déployée comporte quatre étapes.

- La première étape a consisté à concevoir des scénarios de règles de partage de l'eau entre agriculteurs. Chaque scénario repose sur un principe de justice dominant. Il a fait l'objet d'une formulation courte, destinée à être présentée aux agriculteurs lors de l'entretien.

- La seconde a consisté en la réalisation d'entretiens auprès des agriculteurs, choisis dans cinq terrains d'étude (voir figure 1). Dans ces terrains, l'irrigation est principalement dépendante d'eaux souterraines concernées par la gestion quantitative : des quotas individuels de prélèvement sont déjà ou seront prochainement mis en place. Nous sommes entrés en contact avec les agriculteurs par l'intermédiaire de leurs représentants (Chambres d'agriculture, syndicat mixte d'hydraulique agricole, groupements d'agriculteurs). Compte tenu du nombre restreint d'agriculteurs irrigant à partir des nappes souterraines (plaine alluviale du Tarn-et-Garonne par exemple) et/ou des difficultés à mobiliser les agriculteurs (plaine de l'Est lyonnais par exemple), il n'a pas été possible de réaliser d'échantillonnage au sens statistique. Cela constitue la limite de la représentativité de nos résultats, mais nos objectifs initiaux (combiner différents cadres d'analyse et proposer une méthode d'évaluation de l'acceptabilité) n'en sont pas affectés.

Pour chaque scénario, il était demandé à la personne de préciser si elle trouvait ce scénario globalement acceptable, et de donner les raisons de son choix. Sur les 76 agriculteurs interrogés, 29 ont refusé

Figure 1. Localisation et particularité des cultures irriguées des terrains d'étude

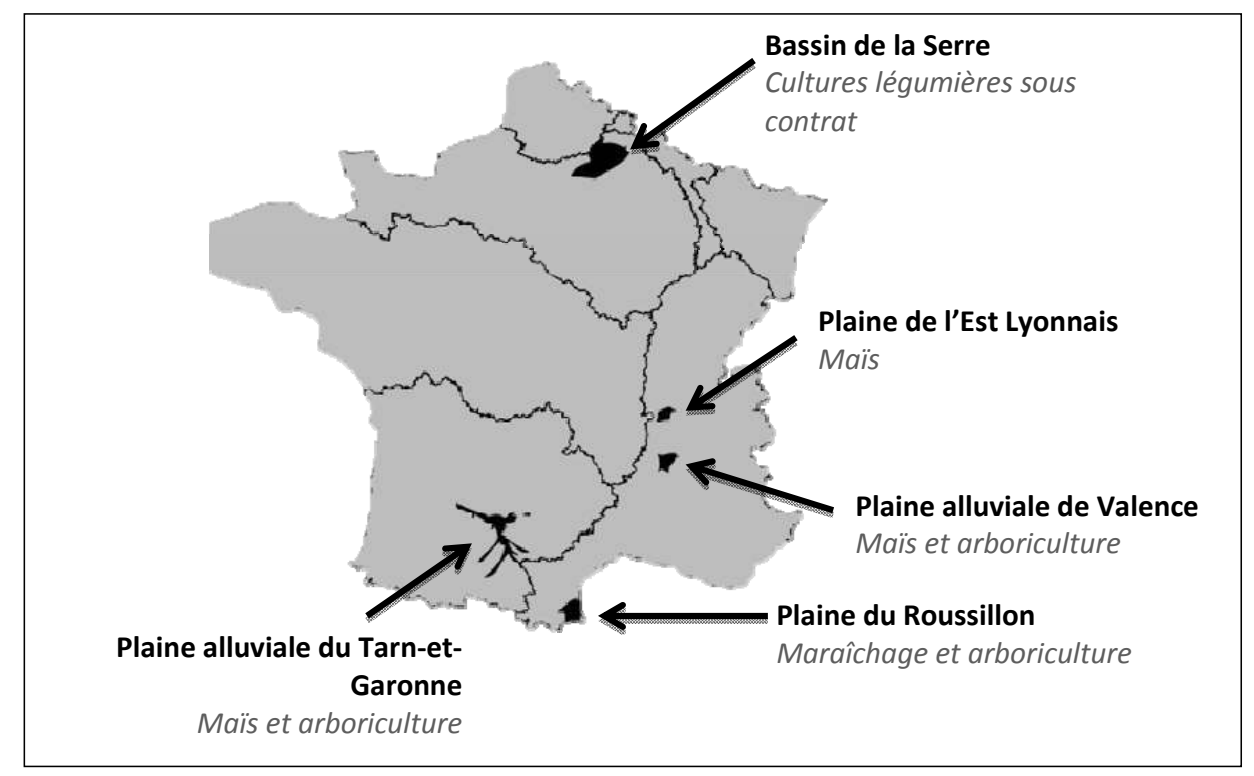




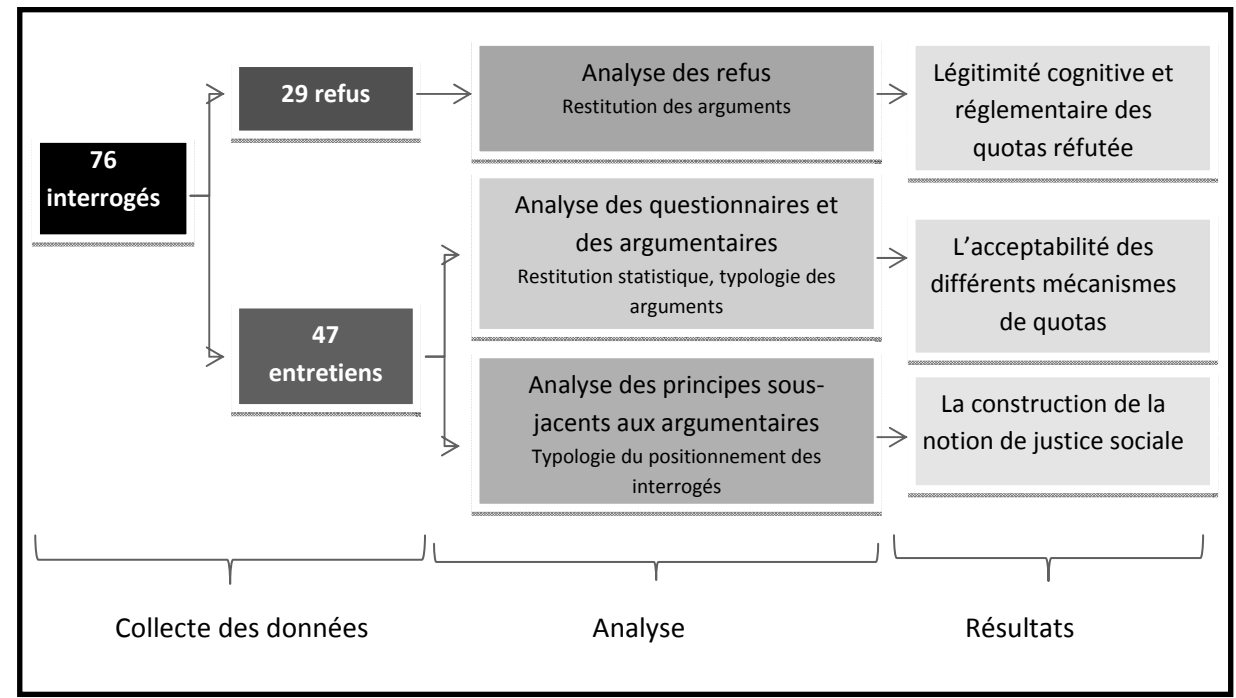

Source : les auteurs.

de nous répondre ${ }^{1}$, et 47 ont accepté de réaliser l'entretien. Parmi les 47 entretiens réalisés, 17 ont eu lieu par téléphone et 30 en face-à-face. Ils ont duré en moyenne 45 minutes (figure 2).

- La troisième étape a consisté en une analyse qualitative des arguments et en une analyse quantitative des préférences exprimées pour les scénarios. Les arguments évoqués ont été retranscrits de façon littérale, puis classés en fonction des principes sous-jacents aux différentes visions de la justice sociale, des critères de légitimation et d'utilisabilité des modes d'allocation proposés.

- La quatrième étape a été consacrée à la restitution des résultats sous forme d'une plaquette et d'une présentation orale sur chaque terrain.

1. Les refus ont souvent été longuement argumentés par les agriculteurs concernés lors de l'entretien téléphonique de prise de contact. Les arguments avancés ont fait l'objet d'une analyse sous l'angle de la légitimation cognitive.

\section{Les mécanismes d'allocation}

Nous avons proposé neuf mécanismes d'allocation d'eau souterraine entre les agriculteurs, tous sous-tendus par un ou plusieurs principes de justice sociale.

- L'allocation est proportionnelle à la consommation des 5 dernières années. Cette règle associe le principe d'antériorité d'usage et le principe de besoin. Ce principe est mis en œuvre dans de nombreuses zones en France.

- L'allocation est ouverte à tous les agriculteurs, irrigant actuellement ou ayant le projet d'irriguer dans les 5 ans à venir. Il s'agit de garantir le principe de stricte égalité d'accès aux ressources. Ce système est en vigueur dans le bassin de la Serre et dans la plaine du Tarn-etGaronne, avec un projet annuel.

- Le volume prélevable est mis aux enchères. L'objectif est de maximiser la valeur marginale de l'eau, satisfaisant ainsi au principe d'efficacité économique. Cette logique existe dans les pays autorisant 
les échanges marchands de droits d'eau (ou quotas) notamment en Australie, en Espagne, au Chili et aux États-Unis.

- L'allocation est réalisée selon l'ancienneté de l'usage, avec priorité aux plus anciens. Cette logique émane directement du principe d'antériorité d'usage, qui garantit l'accès à l'eau aux premiers irrigants. Elle est en vigueur dans plusieurs États de l'Ouest américain.

- L'allocation est proportionnelle au débit déclaré des installations de prélèvements. Ce système est en vigueur dans la plaine du Tarn-et-Garonne. Il permet d'articuler le principe de besoin, évalué par la capacité en débit des équipements en place, et le principe de mérite, car il récompense les agriculteurs ayant correctement déclaré leurs équipements et pénalise ceux qui prélèvent illégalement.

- L'allocation est dégressive en fonction de la superficie irriguée. Le volume par hectare est supérieur pour les exploitations ayant une faible superficie irriguée, afin de leur permettre de dégager plus de valeur ajoutée par unité de surface pour assurer leur viabilité économique. Cette règle est inspirée du principe d'égalité des chances.

- L'allocation dépend du type de production, avec priorité aux cultures spéciales. Ce principe vise à maximiser le revenu que la société tirerait de l'eau, répondant au principe d'efficacité économique. Cette logique est en vigueur dans certains départements français ou les cultures à forte valeur ajoutée peuvent être exemptées de restriction d'arrosage.

- L'allocation tient compte du type de sol. Il s'agit d'attribuer un quota supérieur aux agriculteurs travaillant des sols ayant une faible réserve utile, le besoin en irrigation étant supérieur sur ces terres. Tout en répondant au principe de besoin (agronomique) spécifique, ce scénario satisfait aussi le principe d'égalité des chances. C'est un principe souvent retenu, par exemple sur le bassin de la Serre ou dans l'Eure-et-Loir.

- L'allocation tient compte de l'accès aux eaux de surface. L'eau souterraine est attribuée en priorité à ceux qui n'ont accès à aucune autre ressource ; ceux qui ont accès aux eaux de surface (rivière, retenues) sont appelés à utiliser ces ressources en priorité. Cette règle veut répondre au principe d'égalité des chances, si l'on admet l'hypothèse (forte) que ce qui est déterminant dans l'option eau de surface/eau de nappe n'est pas un choix délibéré des agriculteurs, mais une inégalité de la nature (localisation, topographie). Cette logique est retenue sur la nappe de l'Est lyonnais.

\section{L'acceptabilité des règles de partage et le rôle des arguments éthiques}

\section{Un défaut de légitimation cognitive}

Les 29 personnes qui ont refusé d'évaluer les scénarios ont motivé leur refus selon quatre registres :

- La plupart des réponses remettent en cause la légitimité cognitive des quotas, en affirmant que ce mécanisme véhicule une représentation de la réalité qui n'est ni compréhensible ni acceptable. En effet, certains agriculteurs réfutent la véracité de la pénurie ; ils affirment que l'eau est plus abondante dans leur zone que ne le disent les experts et qu'il n'est pas nécessaire de restreindre les prélèvements. D'autres critiquent la réponse qui est apportée à ce problème, en arguant que l'agriculture est sacrifiée au profit d'autres usages, ou que la société devrait créer de nouvelles ressources (barrages, retenues collinaires).

- Certains réfutent la légitimité réglementaire du principe des quotas. Selon leur argumentaire, les quotas introduiraient une rigidité entravant leur liberté d'entreprendre. Par ailleurs, la restriction 
remet en cause des droits considérés comme acquis, ce qui est estimé comme une violation du droit de propriété (certains invoquent des arguments proches du principe de l'antériorité d'usage). Enfin, la légitimité de l'Administration à intervenir dans la définition des quotas individuels est remise en question, et le risque d'ingérence des problématiques environnementales au sein de celles strictement agricoles est soulevé, comme ce fut le cas dans d'autres études sur des thématiques proches (Aspe, 2012).

- La démarche de consultation entreprise par l'équipe de recherche est récusée par certains agriculteurs qui craignent d'être instrumentalisés et de participer à un « simulacre de démocratie » (Barbier, 2005).

- D'autres, enfin, refusent ou éprouvent des difficultés à adopter une démarche prospective, jugeant l'horizon temporel trop éloigné.
L'opposition a été exprimée de manière « radicale, mais robuste » (Barbier, 2005). En effet, malgré ce refus de discuter des scénarios, les agriculteurs s'inscrivent comme partie prenante de notre réflexion : ils interrogent, au-delà de tout débat sur le mode de calcul des quotas, le bien-fondé du rationnement en eau des aquifères. Ainsi, pour qu'une règle soit acceptable, il faut qu'elle renvoie à une réalité partagée et qu'elle soit mise en place par une institution légitime. L'analyse des argumentaires montre que ces conditions ne sont pas réunies pour les agriculteurs qui ont refusé de nous répondre.

\section{Acceptation des scénarios par les agriculteurs consultés}

La figure 3 permet d'illustrer les principes partagés par tous les terrains et les spécificités des sites, bien que les effectifs soient faibles, avec 47 questionnaires complets au total.

Figure 3. Réponse à la question : "Ce scénario vous paraît-il globalement acceptable ? " (Pour chacun des 9 scénarios, par terrain ou sur l'échantillon complet)

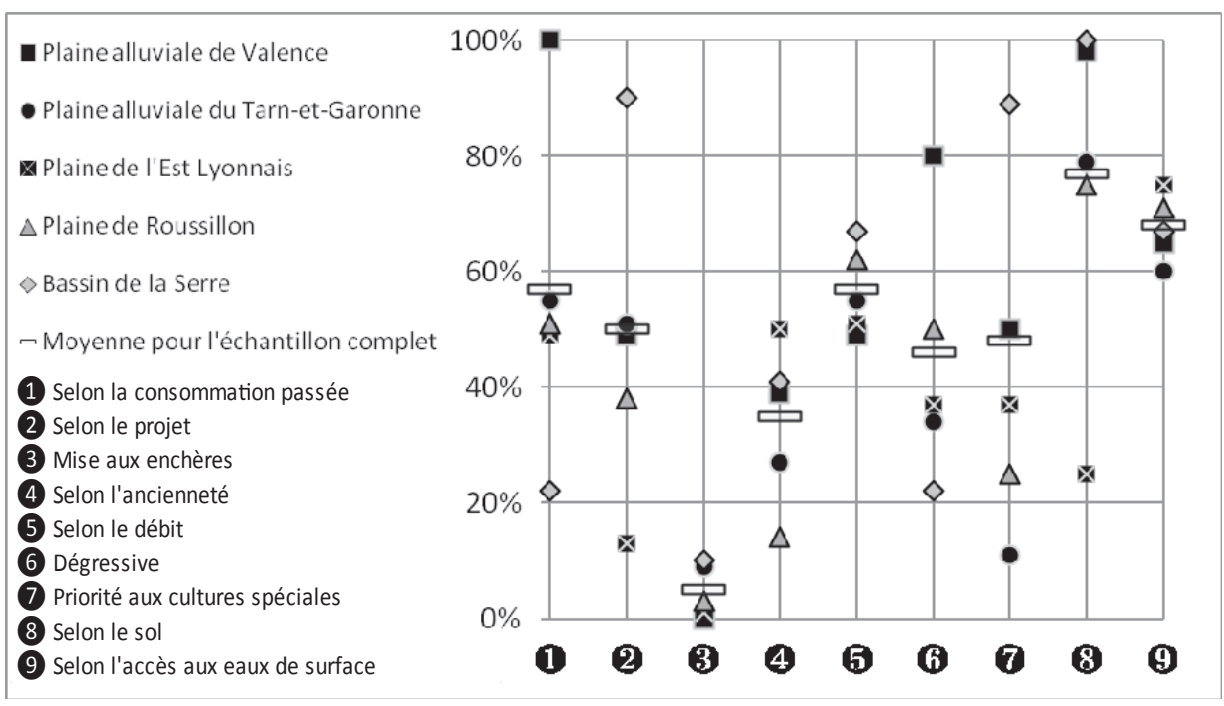

Source : les auteurs. 
Pour quatre des scénarios, la distribution des réponses dépend peu du contexte. Les allocations compensant des inégalités naturelles (selon le type de sol ou selon l'accès aux eaux de surface) ont le taux d'acceptabilité le plus élevé, sauf quand elles sont en inadéquation avec la situation locale (sur la plaine de l'Est lyonnais, les sols sont vus comme trop homogènes pour qu'une différenciation fasse sens, par exemple). À l'inverse, les logiques inspirées de modèles anglo-saxons n'ont suscité l'adhésion que de 16 (priorité aux anciens) et 2 (la mise aux enchères) des 47 répondants.

Pour les autres scénarios, la distribution des avis est très différente selon les terrains. L'allocation basée sur la consommation passée est acceptée par tous les agriculteurs de la plaine de Valence, mais seuls 2 des 9 agriculteurs du bassin de la Serre l'ont validée (en raison de la variabilité interannuelle des précipitations). Le scénario basé sur le projet d'irrigation reçoit à l'inverse l'adhésion des agriculteurs du bassin de la Serre, car il est proche de ce qui s'y pratique déjà, mais il est rejeté par 8 des 9 agriculteurs de la plaine de l'Est lyonnais, où la dynamique actuelle de réduction des surfaces irriguées rend peu pertinente la logique d'ouverture à de nouveaux irrigants. L'allocation avec priorité aux cultures spéciales reçoit l'adhésion de la plus grande partie des interrogés dans la plaine de Valence, les agriculteurs étant sous contrat avec les industries agroalimentaires, tandis que dans la plaine du Tarn-et-Garonne, ce scénario est rejeté, car des tensions existent déjà entre les arboriculteurs et les agriculteurs qui pratiquent les grandes cultures (la préservation de la paix sociale est jugée prioritaire sur un éventuel bénéfice en termes d'allocation de l'eau).

\section{Les facteurs d'acceptabilité}

Quatre facteurs principaux d'acceptabilité se dégagent des argumentaires :
- Éthique et inégalité. Sont regroupés ici les arguments portant sur les valeurs morales qui fondent ces mécanismes. Ainsi, le scénario de mise aux enchères suscite une forte réticence éthique reposant sur le rejet du principe de marchandisation de l'eau. Le scénario d'allocation dégressive provoque des réactions soit favorables en référence à un principe de solidarité, soit défavorables lorsqu'il est assimilé à une logique d'assistanat. Certains agriculteurs soulèvent le risque que les scénarios créent de nouvelles inégalités (la référence aux consommations passées pénaliserait les agriculteurs déjà économes en eau) ou renforcent les inégalités existantes (la priorité à l'ancienneté de l'irrigation qui pénaliserait les jeunes qui sont en pleine phase de remboursement d'emprunts). À l'inverse, une différenciation selon les sols est décrite comme pouvant pallier légitimement une inégalité naturelle subie.

- Mise en cuvre, facilité d'apprentissage et d'appropriation. Il s'agit d'arguments liés à l'application concrète du scénario sur le territoire. Ainsi, un scénario peut être accepté dans son fondement éthique, mais se retrouver invalidé en raison d'une application jugée trop coûteuse ou trop complexe. Le scénario de différenciation de l'allocation selon les sols l'illustre : presque unanimement validé dans son principe, il provoque souvent le scepticisme quant à sa mise en place (négociations longues et conflictuelles pour la classification des parcelles, terrains où le sol est très fortement hétérogène comme dans la plaine du Tarn-et-Garonne, où différents types de sols peuvent se côtoyer sur une même parcelle agricole). Ces critères font donc directement référence aux notions de "facilité d'apprentissage » et « facilité d'appropriation », définis par Nielsen (1994).

- Efficience et effets induits. Ces arguments concernent les conséquences 
qu'aurait un tel système sur les exploitations, sur la structure agricole ou l'économie de la région. Par exemple, le scénario dégressif aurait pour conséquence de diminuer la performance agricole de la région, tandis que la mise aux enchères encouragerait la monoculture. En d'autres termes, dans les discours des interrogés, l'efficience du mécanisme (Nielsen, 1994) ne doit pas se mesurer à l'aune des seuls enjeux de l'eau, mais plus largement au regard des objectifs de développement du territoire.

- Dérives potentielles et fiabilité. Ces arguments concernent le risque du détournement d'un système, qui l'éloignerait de son objectif initial. Cette dimension est spontanément évoquée, en référence à de nombreuses expériences de détournement d'instruments de politiques agricoles (droits paiements uniques, quotas laitiers, remembrement agricole et estimation foncière due au TGV sur la plaine de Valence, etc.). Ainsi, une allocation dégressive selon la surface, censée encourager les petites exploitations, verrait les grandes sociétés se diviser en petites entités. Une allocation avec priorité aux cultures spéciales pourrait donner lieu à des détournements de volumes, qui seraient utilisés pour d'autres types de culture. La notion de « fiabilité » du processus (Nielsen, 1994) est donc essentielle dans le discours des interrogés.

Ces quatre types de facteurs sont spontanément évoqués par les agriculteurs, démontrant ainsi leur habitude d'évaluer, $a$ priori, les politiques agricoles.

\section{Le jugement d'acceptabilité comme articulation entre principes éthiques, d'utilisabilité ou autocentrés}

La dimension éthique est essentielle dans les jugements exprimés, puisque les scénarios qui ont provoqué un jugement éthique unanimement favorable sont plébiscités, et inversement. Cependant, les agriculteurs n'évoquent pas toujours explicitement une théorie ou un idéal de justice pour valider ou invalider une règle de partage. La première réaction est de type affectif, et il n'est pas toujours possible de l'expliciter : AGR41 «Je ne saurais pas comment vous l'expliquer, mais ce scénario, je ne le sens pas. » Nous avons donc dû nous livrer à un travail analytique pour savoir comment les agriculteurs ont statué sur les mécanismes proposés. Les argumentaires montrent une grande diversité de points de vue, dans la place accordée aux arguments éthiques et dans leur nature. Nous avons identifié six grandes logiques que chacun des agriculteurs a mobilisées parfois sans changement de cap tout au long de l'entretien, souvent de manière composite et variable d'un scénario à un autre, conformément à la théorie de l'intuitionnisme. En outre, le poids du contexte local et la dimension stratégique des interrogés ne doivent pas être négligés.

Trois logiques d'acceptabilité basées sur la justice sociale

- La logique d'efficacité économique AGR38 : «En cas de crise, on doit diminuer l'irrigation des céréales pour irriguer les cultures sous contrat. Le plus important, c'est de garantir les cultures avec une forte valeur ajoutée. »

Selon cette logique, l'eau doit être attribuée de façon à maximiser sa valeur et à protéger la sécurité des irrigants : plus l'eau est rare, plus elle a de la valeur, il est donc logique de l'attribuer aux cultures qui dégagent le plus de revenus (par exemple les cultures sous contrat). À l'inverse, l'allocation dégressive est taxée de «trop sociale » et contraire à la logique d'efficacité.

- La logique de stricte égalité AGR28 : «J'aime pas l'idée de faire des différences entre les Blancs et les Noirs, les petits et les gros. » 
Cette logique implique d'appliquer le même coefficient de restriction à tous les irrigants, en se basant sur la consommation passée, le projet d'irrigation ou le débit d'équipement. Cela permet à la fois de ne pas créer de nouvelles inégalités et d'être facile à mettre en place. Cela est d'autant plus important en contexte de crise : AGR28 «Le plus juste, c'est de déstabiliser le moins possible le système économique avec les restrictions, il vaut mieux enlever pareil à tous. » Cette logique invalide tous les systèmes fondés sur une pondération (mise aux enchères, l'allocation selon l'ancienneté, l'allocation dégressive).

- La logique d'égalité des chances

AGR17 : «Je suis pas socialiste, mais je suis social. Il faut que tout le monde fasse des efforts, mais selon ses capacités. Il ne faut pas non plus des distorsions trop fortes, tout le monde a le droit de vivre. »

Selon ce positionnement, l'effort consenti par tous n'est pas forcément égal, et il doit prendre en compte la situation, les caractéristiques ou les difficultés de chacun, dans une perspective d'égalité des chances. La politique d'allocation de la ressource en eau s'apparente alors à un mécanisme de redistribution sociale (références dans les discours à l'allocation-chômage, au système des retraites, au droit au logement). Un coefficient de restriction unique risquerait d'entériner les inégalités existantes (AGR11 «Quelqu'un qui part hors cadre familial, il est défavorisé, et c'est plus compliqué aussi financièrement. Il ne faut pas le léser. »), voire de créer de nouvelles inégalités.

- La logique procédurale collective AGR45 : «C'est compliqué de s'entendre, mais on n'a pas le choix. Dans les années 1960, on nous a forcés à être individualistes, mais on ne peut pas faire l'économie de raisonner en collectif. »
Cette conception de la justice sociale est basée sur deux notions inséparables ici : d'une part, sa dimension procédurale dans la manière de définir collectivement le partage de l'eau et d'autre part, la dimension sociétale du projet territorial dans lequel s'insère l'irrigation. Selon cette logique, ce système des quotas ne doit pas se résumer à un droit individuel à produire ; et le calcul des quotas ne doit pas être le résultat d'une négociation entre les exploitants ou les lobbies. Ce doit être l'occasion d'un débat de société ; et la politique d'allocation doit être un levier d'une politique territoriale et agricole. La vision doit donc être élargie en termes de thématiques, d'espace et de temps : AGR32 «Ce système est inverse au développement durable, qui permet à ceux qui viennent après vous de vivre mieux que vous. C'est ce que moi j'appelais gestion "en bon père de famille”, c'est-à-dire l'inverse de "comment je peux me protéger, moi ?" » Cette logique définit l'agriculteur non pas comme un propriétaire du droit d'eau, mais comme son dépositaire, dans la lignée des travaux d'Elinor Ostrom (1990) d'une gestion de l'eau pouvant s'inspirer des principes de gestion des ressources en accès commun, qui peut s'opposer efficacement à une gestion par le marché ou par l'État. La référence à l'Association syndicale autorisée (ASA) est utilisée par les agriculteurs pour valider cette logique, en tant qu'exemple concret de gestion collective des périmètres irrigués.

\section{Critère d'utilisabilité au cœur de l'acceptabilité}

- La logique de l'efficacité agronomique AGR20 : «Ce que vous proposez, ça ne me plaît pas, c'est une gestion sociale et pas agronomique. L'eau, ce n'est pas social, c'est agronomique. »

Si ces agriculteurs ne remettent pas forcément en cause la réalité de la pénurie et la nécessité de la restriction, ils s'opposent à ce que cette restriction se fasse sur des critères autres que des paramètres 
agronomiques (besoin de la plante, sol, pluviométrie, etc.). Toute logique alternative constitue une intrusion des problématiques sociales ou économiques au sein d'une problématique d'efficience technique. Les seules allocations valables reposent sur le type de sol, l'allocation selon la consommation passée, et le débit d'équipement, car reflet des besoins en eau des cultures. Un scénario nous a été suggéré en plus de ceux que nous avions proposés : une allocation basée sur le besoin théorique des cultures, associé si besoin à un coefficient de restriction unique. Ce positionnement remet en cause la façon dont nous avons posé les termes du débat, en présupposant que la répartition de l'eau pose des enjeux de justice sociale.

\section{- Une lecture autocentrée}

AGR10 : "Dans votre questionnaire, chacun va choisir la solution qui l'arrange le plus. »

Certains agriculteurs ont étudié les mécanismes proposés un par un, indépendamment d'une référence explicite à une logique de justice ou à des critères d'utilisabilité, mais, selon l'intérêt et le danger qu'il présente à titre personnel : AGR22 «Je suis un des plus vieux irrigants de la région, je vais prêcher pour ma paroisse. » Ce mécanisme de subjectivité dans l'évaluation du caractère juste ou injuste d'une option quand l'individu est directement affecté par la situation de choix est qualifié de fairness bias (Johansson-Stenman et Konow, 2010). Au-delà des agriculteurs qui ont résolument revendiqué cette posture pendant tout l'entretien, ce fairness bias a pu interférer dans le jugement des autres interviewés.

Pour conclure, on peut faire le lien entre les six principes de justice identifiés initialement dans la littérature et les logiques mobilisées lors des entretiens. Trois principes de justice sont reconnus comme déterminants par les interrogés : l'efficacité, l'égalité des chances et la stricte égalité, alors que le principe d'antériorité d'usage est rejeté. Le principe de besoin est identifié comme essentiel par une partie des interrogés ; mais est formulé comme un principe de gestion agronomique (besoin en eau de la plante) et non comme un principe de justice sociale. Enfin, le principe de mérite a été évoqué, mais associé à d'autres principes : l'efficacité économique se base en partie sur la notion de mérite (reconnaissance des efforts réalisés par les exploitants les plus performants) et le rejet du principe d'antériorité d'usage se base sur la reconnaissance de l'investissement de chaque exploitant.

\section{La justice situationnelle comme intrication des enjeux éthiques et stratégiques}

Pendant l'entretien, les interrogés se sont positionnés par rapport aux principes de justice en les validant, les rejetant ou en les combinant. Dans le même temps, ils se sont positionnés par rapport aux enjeux économiques et sociaux que représente la mise en place des quotas. Les risques de dégradation de leur situation personnelle, de conflits sociaux entre les différents groupes d'agriculteurs ou avec l'administration ont été évalués par les agriculteurs et ont guidé leur réponse, parfois de façon implicite, parfois de façon explicite. Cette dimension stratégique a pu être renforcée par la perception que les agriculteurs ont eue de notre enquête (émanation directe des institutions ou première étape de la mise en place des quotas).

Notre analyse ne différencie pas, dans le discours des interrogés, ce qui relève de leur conviction sur les règles de partage de l'eau et ce qui tient à un positionnement stratégique dans le jeu d'acteurs. En effet, nous considérons que ces deux types d'enjeux sont intriqués, conformément à la théorie de la justice situationnelle, qui comprend à la fois la défense 
de ses principes moraux, la défense de ses propres intérêts et des jugements sur la situation des autres personnes affectées (Syme et al., 1999). En outre, l'enjeu pour les Organismes uniques sera de prendre en compte ces différences de positionnements éthiques, tout en considérant les jeux d'acteurs qui peuvent exister sur leur territoire.

\section{Discussion}

\section{Validation du cadre d'analyse général}

Notre cadre d'analyse a été validé : nos résultats montrent que l'acceptabilité d'une règle peut être définie par quatre dimensions : i) sa légitimité cognitive, c'est-à-dire le système symbolique véhiculé, qui doit renvoyer à une réalité compréhensible et acceptable, ii) sa légitimité réglementaire, c'est-à-dire que la règle et les institutions qui la portent doivent s'inscrire dans le cadre des régulateurs explicites que sont les lois, les contrôles et les sanctions, iii) sa légitimité normative, c'est-à-dire la cohérence entre les valeurs éthiques de la règle et celles de la société et enfin iv) son utilisabilité, c'est-à-dire la capacité de la règle à être mise en place sans heurter les pratiques et les ressources des usagers.

Dans nos cas d'études, l'importance des critères éthiques est fonction des principes qui les sous-tendent. Réduire l'eau à sa dimension économique à travers les marchés de l'eau et protéger les plus anciens irrigants au prétexte qu'ils auraient pris des risques en tant que pionniers de l'irrigation sont des principes quasi unanimement réfutés pour des raisons de justice sociale, quel que soit le terrain. Ces deux critères (le principe d'efficacité économique poussé jusqu'au droit du plus offrant et le principe d'antériorité d'usage) sont aussi le plus souvent rejetés dans les enquêtes de Nancarrow et Syme (2004) en Australie.
Sur les territoires étudiés, dans un idéal délibératif, atténuer les inégalités naturelles au sein de chaque zone afin d'égaliser les chances entre irrigants (diversité des sols, de l'accès à l'eau de surface) tout en reconnaissant la diversité des besoins identifiés (reflétés par le débit d'équipement et la consommation passée), puis gérer solidairement la pénurie, sont des valeurs partagées par un grand nombre d'agriculteurs. Des critères liés à l'efficacité économique (protection des cultures spéciales, marchés) ou à la protection des plus vulnérables (allocation dégressive) soulèvent plus de réticences et polarisent plus fortement les positionnements des agriculteurs.

Deux aspects importants révélés par nos travaux sont absents dans le travail des équipes de recherche australiennes. Premièrement, nos interrogés soulèvent le manque de légitimité cognitive et réglementaire de cette gestion de la rareté de l'eau de nappe, perçue comme une rareté administrative et non naturelle en France. Deuxièmement, les argumentaires révèlent l'importance des considérations pragmatiques dans le jugement d'acceptabilité des règles de partage. Quatre des cinq critères d'utilisabilité de Nielsen (1994) à savoir l'efficience, la facilité d'apprentissage et d'appropriation, la fiabilité sont des éléments souvent prépondérants dans le jugement des interrogés et peuvent invalider toute pertinence de considérations éthiques pour gérer la ressource. En outre, quand les interrogés ont passé en revue les scénarios d'allocation, nombre d'entre eux empruntaient des argumentations relevant tantôt de la justice sociale, tantôt de l'utilisabilité. Leur jugement a pu être aussi influencé par l'évaluation, que chacun a faite, des conséquences concrètes que cette règle aurait sur sa propre exploitation. Statuer sur l'incohérence et la volatilité de ces jugements serait pourtant une erreur. Il s'agit de constater le besoin de composer 
et d'articuler différentes façons de considérer le juste au sein de la plupart des individus, dans la lignée des travaux de Konow sur l'intuitionnisme de sens commun (Schaeffer et Aubert, 2010). La différence entre les terrains, avec des convergences locales fortes des adhésions sur certains principes révèle que les conséquences de certaines options sur des dynamiques de développement agricole ont été mesurées par les interrogés, de même que les incompatibilités avec des trajectoires historiques ou des caractéristiques du milieu.

\section{Conséquences pour la gestion de l'eau}

Au-delà des aspects éthiques et de paix sociale, la légitimation du dispositif est un critère pragmatique, essentiel à la durabilité des systèmes irrigués gérés par les collectifs concernés. Selon Ostrom (1992), une institution peut gérer durablement un système irrigué si elle présente pour les usagers une incitation, qui peut être matérielle ou symbolique (sentiment d'appartenance, confort personnel dans les relations sociales...), qui repose sur les valeurs partagées des individus.

En France, les politiques publiques s'orientent vers un transfert de la gestion de la ressource en eau aux usagers, représentés ici par les Organismes uniques. Cette dynamique est à replacer dans un contexte de faiblesse de moyens de la police de l'eau et de la multitude des points de prélèvement dans les nappes. Or les Organismes uniques, déjà fragiles en raison de leur caractère récent et de la faiblesse des moyens financiers alloués aux processus de régulation et contrôle de l'accès à l'eau, vont devoir affronter les défauts de légitimité cognitive et institutionnelle, préalable à l'instauration des règles de partage.

Le défaut de légitimité cognitive des quotas, s'exprime par les réticences à entériner le diagnostic de rareté de la ressource et à accepter les restrictions qui en découlent. Il résulte du « hiatus entre expertise scientifique, gestionnaire, et observation profane sur l'état du milieu naturel et les prescriptions technico-environnementales » (Barbier, 2005). Ces agriculteurs qui vont jusqu'à refuser de discuter des principes de justice ne nient pas l'existence de régions en tension sur la ressource, mais ils réfutent ce constat pour leur propre territoire. Ce déni d'une traduction locale d'un enjeu largement médiatisé au niveau national rejoint celui de l'analyse de Salles (2006) sur les risques de pollutions diffuses agricoles autour de périmètres de captage d'eau potable dans le Sud-Ouest : «Ils estiment que leur territoire est à l'abri des dérives observées dans d'autres régions » (Salles, 2006). En outre, ces réticences traduisent un positionnement stratégique de certains agriculteurs. Quelques-uns ont pu voir dans notre travail de recherche un moyen d'infléchir les décisions futures en suggérant une situation peu problématique. D'autres, comme ceux qui ont refusé de répondre ont pu craindre au contraire d'accélérer le processus administratif s'ils validaient un état de tension sur l'eau souterraine.

La résistance aux principes des quotas s'adosse aussi à un défaut de légitimation réglementaire, c'est-à-dire à l'absence de conformité entre une règle et l'ensemble des régulateurs explicites que sont les lois, les contrôles et les sanctions (Scott, 2000). En effet, certains agriculteurs remettent en cause la légitimité des institutions, ou le manque de cohérence de leurs actions : AGR65 «La VNF ${ }^{2}$ nous donne une première autorisation. La DDT ${ }^{3}$ une deuxième autorisation. Et si c'est une ravine, on a une autre autorisation. Ça fait trois autorisations différentes, pour la même eau!» La représentativité de certaines institutions est critiquée : AGR68 «Dans les ASA, ils

2. Voies navigables de France.

3. Direction départementale des territoires. 
ne veulent pas d'agriculteurs, c'est trop contestataire pour eux. » En outre, le principe même de régulation de l'eau est critiqué, au nom de la liberté d'entreprendre ou de la propriété privée. Ce déficit de légitimité réglementaire implique une difficulté réelle à débattre des questions éthiques liées aux quotas. En effet, pour qu'un débat en termes de justice sociale puisse avoir lieu, cela suppose un cadre institutionnel bien constitué : «Le jugement sur une répartition juste des droits et obligations n'est pas séparable de l'équipement institutionnel d'une communauté politique dont la constitution préalable est nécessaire pour instituer un espace commun de délibération et d'épreuves au milieu duquel la question du juste peut émerger, progresser et aboutir »(Godard, 2004). On rejoint ici des considérations sur la dimension procédurale de la justice d'une réforme, largement étudiée par Nancarrow et Syme dans le cas australien, à côté des critères de justice distributive. Si nous avions délibérément choisi de ne pas aborder la justice procédurale, les agriculteurs ont parfois bousculé ce cadre imposé, discutant la légitimité des institutions à porter la gestion de l'eau agricole AGR54 «On gère l'eau comme on sait le faire, c'est pas un gars dans un bureau qui va nous dire ce qu'on doit faire. »

Dans un contexte difficile, on peut faire l'hypothèse que les responsables des Organismes uniques privilégient une modalité d'allocation proche de la situation actuelle, afin de ne pas perturber les positions acquises qui caractérisent les historiques de prélèvements et d'asseoir ainsi leur légitimité. Le quota, outil administratif, se rapprocherait ainsi du « disponible naturel » de la nappe qui limiterait « naturellement » les prélèvements en l'absence de régulation réglementaire. L'approbation sur la plupart des terrains de l'allocation selon l'historique de consommation laisse augurer ce choix : AGR15 «Ce système paraît simple, ça se fait souvent comme ça dans le monde agricole. » ; «Ça pourrait passer. Mais c'est pas forcément juste, entre celui qui ne fait pas d'efforts et celui qui en a fait beaucoup... » Dans ce cas d'étude comme dans d'autres études portant sur la mise en place d'une gestion quantitative de la ressource en eau (Mazeaud, 2011), il semble difficile de s'émanciper de la « dépendance au sentier », qui implique une continuité dans les choix et dans les pratiques, afin de conforter les savoir-faire et les rentes. Seul le bassin de la Serre fait exception, puisque les agriculteurs se sont saisis de ces questions, la Chambre d'agriculture ayant elle-même financé une étude sur le niveau de la nappe.

Ailleurs, cette légitimation fait courir deux risques. Le premier est de ne pas resituer le débat sur le calcul des quotas dans une réflexion plus profonde, avec la justice sociale comme référent possible, en plus de l'utilisabilité. Le deuxième risque est de voir un durcissement des positions autour des intérêts individuels ou de filière. En fin d'entretiens, nous avons observé plusieurs fois des crispations autour des avantages personnels, ou des craintes que la mise en place de quotas ne donne lieu à des conflits violents : AGR 3 « Je suis contre la restriction, et vous me faites des propositions, alors que je suis pas d'accord sur tout. C'est des trucs à provoquer la guerre. Si on veut provoquer la guerre, je préfère les cultures spéciales, parce que ça m'arrange, moi. » Dans cette perspective où l'évaluation d'un mode de calcul est vécue comme un rapport de force entre les acteurs du territoire, les questions de justice risquent d'être évincées par les jeux de pouvoir, et la gradation en termes de jugement sur la justice laissera place à la hiérarchie sociale ou économique. Cette hypothèse va dans le sens des conclusions de deux études menées en Angleterre et en Australie sur les quotas de pêche (quotas de rejet de prises et quotas de pêche à la 
langouste) : les solutions retenues n'étaient pas les plus justes, mais celles qui ne remettaient pas en cause les asymétries de pouvoir en place (Gray et al., 2011 ; Presser, 1994).

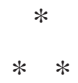

Cette étude propose une mise en perspective de deux types d'approche : l'évaluation de l'acceptabilité d'une politique de partage de l'eau sur un territoire, et l'étude des principes de justice sociale sous-jacents aux argumentaires.

Trois niveaux d'acceptabilité se dégagent ; ils traduisent différentes combinaisons entre les notions d'acceptabilité et de justice sociale. Le premier niveau porte sur la concordance des règles avec la réalité physique et agronomique de la parcelle et du bassin versant (hydrographie, besoin en eau des cultures, etc.). À ce niveau, les critères liés à l'utilisabilité sont prégnants, le système de calcul des quotas étant jugé à l'épreuve du terrain : il doit rester efficace, fiable et appropriable dans son utilisation courante, et ce, malgré les contraintes spécifiques de la zone. Le deuxième niveau d'acceptabilité interroge la cohérence de la règle avec l'histoire du territoire, ses filières agricoles, son expérience de la gestion collective (de l'eau, du foncier, ou des quotas laitiers). Dans cette approche, l'acceptabilité de la règle repose sur sa légitimité cognitive, c'est-àdire l'adéquation entre le système de significations de la règle et celle du territoire, mais aussi sur la légitimité réglementaire d'une institution à intervenir sur ces questions. Légitimité réglementaire et cognitive se rejoignent dans l'idée de distance physique, ou de distance de pratiques, entre les acteurs « des bureaux » et ceux « du terrain ». Enfin, le dernier niveau d'acceptabilité concerne l'échelle du monde agricole dans son ensemble, voire de la société française : l'appartenance à cette communauté confère à ses membres un socle de valeurs culturelles et éthiques, qui amènent à trouver d'emblée certains systèmes comme inacceptables, du fait des valeurs qu'ils véhiculent (marchandisation de l'eau par exemple).

Il revient aux Organismes uniques de mettre en place les quotas individuels, et de proposer un mode de calcul qui soit accepté et appliqué. Ces Organismes uniques devront relever trois défis. Le premier défi est celui de l'utilisabilité, en proposant un système de quota dont le coût soit supportable par la communauté, qui soit appropriable par les usagers, et qui prenne en compte au mieux les spécificités du territoire. En cela, le périmètre d'action des Organismes uniques (bassin versant et nappes) peut être un véritable atout. Le deuxième défi est celui de la légitimité : en tant qu'entité administrative nouvelle, et étant donné le contexte difficile de la mise en place de la loi sur l'eau, les Organismes uniques devront s'insérer dans un territoire déjà marqué par une histoire et des expériences partagées. Enfin, le dernier défi est celui de la justice sociale : nos résultats ont montré que les critères éthiques sont une composante majeure de ce qui est déclaré comme acceptable. Cependant, cette dimension ne pourra être prise en compte qu'à la condition d'un vrai débat de société au sein du monde agricole, et qui ne substitue pas à l'impératif de justice la simple prise en compte des rapports de force.

Les auteurs remercient les exploitants agricoles qui ont consacré du temps à cette enquête, ainsi que les Chambres d'agriculture ayant facilité la prise de contact. Cette étude a été réalisée grâce au soutien financier de l'agence de l'Eau RhôneMéditerranée-Corse dans le cadre du projet SIMGDES. 


\section{RÉFÉRENCES BIBLIOGRAPHIQUES}

Alexander S., Ruderman M. (1987). The role of procedural and distributive justice in organization behavior. Social Justice Research, vol. $1, \mathrm{n}^{\circ} \mathrm{n}^{\circ} 2$, p. 177-198.

Aspe C. (2012). De l'eau agricole à l'eau environnementale. Update Science et Technologies, Versailles, Quae.

Barbier R. (2005). Quand le public prend ses distances avec la participation. Natures Sciences Sociétés, n 3, p. 258-65.

Del Corso J.-P., Nguyen G., Képhaliacos, C. (2014). Quelles conditions à l'acceptation d'un dispositif incitatif de politique publique en agriculture ? VertigO - La revue électronique en sciences de l'environnement, horssérie 20.

Dubois M., Bobillier-Chaumon M.-É. (2009). L'acceptabilité des technologies : bilans et nouvelles perspectives. Le travail humain, vol. $72, \mathrm{n}^{\circ} 4$, p. 305.

Figureau A.-G., Montginoul M., Rinaudo J.-D. (2014). Scénarios de régulation décentralisée des prélèvements agricoles en eau souterraine. Évaluation participative dans le bassin du Clain. Économie rurale, $\mathrm{n}^{\circ} 342$, p. 27-44.

Figureau A.-G., Rinaudo J.-D., Montginoul M. (2012). Gestion quantitative de l'eau d'irrigation en France : bilan de l'application de la loi sur l'eau et les milieux aquatiques de 2006. RP-61626-FR, BRGM.

Garin P., Loubier S. (2007). Des associations d'irrigants se réforment en façonnant ce qu'elles trouvent juste et équitable. Ingénieries - EAT, $\mathrm{n}^{\circ}$ 49, p. 27-38.

Godard O. (2004). L'équité dans les négociations post-Kyoto : critères d'équité et approches procédurales. Cahiers de l'école polytechnique, $\mathrm{n}^{\circ} 8$.

Gray T., Korda R., Stead S., Jones E. (2011). Quota discarding and distributive justice: the case of the under-10 $\mathrm{m}$ fishing fleet in sussex, England. Marine Policy, vol. 35, $\mathrm{n}^{\circ} 2$, p. $122-129$.

Johansson-Stenman O., Konow J. (2010). Fair air: distributive justice and environmental economics. Environmental and Resource Economics, vol. 46, $\mathrm{n}^{\circ}$ 2, p. 147-166.

Konow J. (2003). Which is the fairest one of all? A positive analysis of justice theories. Journal of Economic Literature, vol. 41, $\mathrm{n}^{\circ} 4$, p. 1188-1239.

Konow J. (2009). Is fairness in the eye of the beholder? An impartial spectator analysis of justice. Social Choice and Welfare, vol. 33, $\mathrm{n}^{\circ} 1$, p. 101-27. doi:10.1007/s00355-008-0348-2.

Lafitte J.-J. (2008). Les organismes uniques d'irrigation. Paris, Rapport public.

Lamont J., Favor C. (1996). Distributive Justice. In Edward Zalta N., The Stanford Encyclopedia of Philosophy.

Loubier S., Campardon M., Morardet S. (2013). L'irrigation diminue-t-elle en France ? Premiers enseignements du recensement agricole de 2010. Sciences Eaux \& Territoires, vol. 11, $\mathrm{n}^{\circ}$ 2, p. 12-19.

Martin P. (2013). La gestion quantitative de l'eau en agriculture, une meilleure vision pour un meilleur partage. Paris, Rapport parlementaire.

Mazeaud A. (2011). Quel est le bon débit de l'eau ? Les régulations territoriales sur les usages de l'eau à l'épreuve de la DCE. Pôle Sud, vol. 35, n², p. 59-75.

Montginoul M., Rinaudo J.-D. (2011). Quels outils de gestion des prélèvements en eau agricole pour faire face à la réduction annoncée des disponibilités en eau ? Éléments de réflexion à partir de scénarios de prospective discutés avec des agriculteurs et des institutionnels. AgroSup Dijon, $5^{\text {es }}$ Journées de Recherches en Sciences Sociales, InraSfer-Cirad.

Movik S. (2014). A fair share? Perceptions of justice in South Africa's water allocation reform policy. Geoforum, n ${ }^{\circ} \mathrm{n}^{\circ} 54$, p. 187-95.

Mul M., Kemerink J. S., Vyagusa N., Mshana M. G., van der Zaag P., Makurira H. (2011). Water allocation practices among smallholder farmers in the south pare mountains, tanzania: the issue of scale. Agricultural water management, smallholder 
systems innovations for integrated watershed management in Sub-Saharan Africa, vol. 98, $\mathrm{n}^{\circ} 11$, p. 1752-60.

Nancarrow B. E., Syme G. J. (2004). Fairness principles in allocating water: integrating views of different agents." In iEMSs 2004 International Congress, Complexity and Integrated Resources Management. International Environmental Modelling and Software Society, p. 14-17.

Nancarrow B. E., Syme G. J. (2001). Challenges in implementing justice research in the allocation of natural resources. Social Justice Research, vol. 14, n 4, p. 441-52.

Nielsen J. (1993). Usability Engineering. Boston, Academic Press.

Ostrom E. (1990). Governing the commons: The evolution of institutions for collective action. Cambridge MA, Cambridge University Press.

Ostrom E. (1992). Crafting institutions for selfgoverning irrigation systems. San Francisco CA, ICS Press.

Presser J. (1994). Distributive justice in allocating individual catch quota in a fishery. Australian Agricultural and Resource Economics Society, 1994 Conference (38 th), February 8-10, Wellington, New Zealand 148672.
Roa-García M. C. (2014). Equity, efficiency and sustainability in water allocation in the andes: trade-offs in a full world. Water Alternatives, vol. $7, \mathrm{n}^{\circ} 2$.

Salles D. (2006). Les défis de l'environnement : démocratie et efficacité. Paris, Éditions Syllepse.

Schaeffer Y., Aubert F. (2010). La pertinence de la politique rurale à l'aune des théories de la justice. Revue d'études en agriculture et environnement, vol. 91, $\mathrm{n}^{\circ}$ 1, p. 73-101.

Scott W. R. (2000). Institutions and Organizations. 2nd edition, Thousand Oaks CA, Sage Publications.

Syme G. J., Mc Creddin J. A., Nancarrow B. E. (1999). Defining the Components of Fairness in the Allocation. Journal of Environmental Management, $\mathrm{n}^{\circ}$ 57, p. 51-70.

Syme G. J., Nancarrow B. E. (1997). The determinants of perceptions of fairness in the allocation of water to multiple uses. Water Resources Research, vol. 33, ${ }^{\circ} 9$, p. 2143-2152.

Tisdell J. G. (2003). Equity and social justice in water doctrines. Social Justice Research, vol. $16, n^{\circ} 4$, p. 401-416.

Wenz P. S. (1988). Environmental justice. Albany NY, State University of New York Press. 